\title{
Statistical Multiplexing of Homogeneous Streams Results in Linear Bandwidth Gains
}

\author{
Bushra Anjum \\ Department of Computer Science, North Carolina State University, 809 Oval Drive, Raleigh, NC 27695. \\ Email: banjum@ncsu.edu
}

Received 2012

\begin{abstract}
Statistical multiplexing of traffic streams results in reduced network bandwidth requirement. The resulting gain increases with the increase in the number of streams being multiplexed together. However, the exact shape of the gain curve, as more and more streams are multiplexed together, is not known. In this paper, we first present the generalized result that the statistical gain of combining homogeneous traffic streams, of any traffic type, is a linear function of the number of streams being multiplexed. That is, given a fixed Quality of Service (QoS) constraint, like percentile delay, $D$, the bandwidth requirement of $n$ streams to satisfy the delay constraint $D$ is $n \times R \times c$ where $R$ is the bandwidth requirement of a single stream that satisfies the constraint $D$ and c $\varepsilon(0,1]$. We present the linear bandwidth gain result, using an extensive simulation study for video traces, specifically, streaming video (IPTV traces) and interactive video (CISCO Telepresence traces). The linear bandwidth gain result is then verified using analytical tools from two different domains. First, we validate the linearity using Queueing Theory Analysis, specifically using Interrupted Poisson Process (IPP) and Markov Modulated Poisson Process (MMPP) modeling. Second, we formally prove the linear behavior using the Asymptotic Analysis of Algorithms, specifically, the Big-O analysis.
\end{abstract}

Keywords: Quality-of-Service; Bandwidth; Video Traces; IPTV; CISCO Point-to-Point Telepresence

\section{Introduction}

Statistical multiplexing and effective bandwidth requirements have been the focus of many research studies, approximate algorithms and network planning heuristics. However, their main focus remain on utilizing statistical multiplexing to maximize the number of streams a connection carries while also satisfying some QoS measures like delay, jitter and/or packet loss.

In this paper, we focus on the nature of statistical multiplexing itself and try to determine the shape of the bandwidth gain curve as more and more streams, constrained by a single end-to-end QoS measure, are multiplexed together. Hence, our first major contribution is the result that the bandwidth gain of multiplexing homogeneous streams is "linear". This linear behavior came as a surprise as we were expecting atleast a second degree polynomial gain. Our second contribution is that we were able to verify this linear behavior using analytical tools from two different domains. First, using Queueing Theory approach and modeling a single trace with an IPP and multiplexed traces using a single MMPP-n distribution (which is an exact representation of $n$ IPPs multiplexed together).The result was also validated using the analysis of Asymptotic Bounds from the field of Algo- rithms.

The results carry significant importance in the communications networks as they simplify network dimensioning and resource planning. It brings down the problem of allocating bandwidth, for multiplexed traffic streams of the same traffic type, from heuristics and approximations to exact calculations.

Below we give a brief literature survey of the latest work being conducted in the areas of statistical multiplexing, bandwidth gain with special focus on video traffic. Van der Auwera in [1] examines the implications of video traffic smoothing on statistically multiplexed video streams, focused on identifying the levels of smoothing that give (bufferless) statistical multiplexing performance close to an optimal off-line smoothing technique. Angrishi in [2] uses the large deviations theory to express the QoS measure at the link in terms of effective capacity. Li, Huang and Devetsikiotis in [3] work on overcoming the conservative nature of effective bandwidth by using a robust adaptive effective bandwidth allocation algorithm using statistical multiplexing gain occurring among multiple traffic classes. Ravindran and Rabby in [4] describe a policy based model for cost effective data connectivity provisioning involving flow aggregation over a connection. Jeong, Jeon, Jung and Choe in [5] focus on a statis- 
tical multiplexing system that supports layered multicast to provide heterogeneous device capability in the video multicasting/broadcasting. Again, it should be emphasized that the focus of the research presented above is to utilize the statistical gain for improved QoS provisioning and maximizing resources, and not to determine the shape of the gain itself. The later is the focus of our paper and we present the main result that the shape of the gain for homogeneous multiplexed streams is linear.

Rest of the paper is organized as follows. In section 2 we first describ the simulation setup in detail. We then present the required bandwidth results, for Telepresence and IPTV traces, as a function of the number of homogeneous streams so that the end-to-end percentile delay remains the same. In section 3 we verify our results using Queueing theory analysis and further in section 4 using asymptotic analysis. Conclusions are in section 5 .

\section{Bandwidth Requirement for Homogenous Flows}

In this section, we calculate the bandwidth required to satisfy a given QoS delay constraint $D$ as a function of the number of homogeneous video streams $n$. We present the result that given a fixed percentile delay $D$, the bandwidth requirement of $n$ homogeneous streams to satisfy the delay constraint $D$ is $n \times R \times c$ where $R$ is the bandwidth requirement of a single stream that satisfies the constraint $D$ and c $\varepsilon(0,1]$. We can easily calculate the factor $c$ as follows: First we calculate/simulate the bandwidth requirement so that a single stream satisfies the constraint $D$, let us call it $R_{1}$. Next we calculate the bandwidth requirement for any value of $n$ (for $n>3$ ) streams to satisfy the same constraint $D$, let us call the $n$ value $n^{\prime}$ and the calculated bandwidth $R$ '. Then $c$ is simply the multiplicative factor which can be calculated as $n^{\prime} R_{1} / R^{\prime}$. The factor $c$ can then be used to calculate the bandwidth requirement for any value of $n$ for the same delay constraint $D$.

We are using two different types of traces, namely, CISCO point-to-point Telepresence and IPTV. The simulation program was implemented in Java and it simulates a tandem 10-node network, where each node depicts the output port of a router. Our focus is on an MPLS connection that is being allocated specifically for the video traffic under consideration. Since an MPLS connection implies the same bandwidth allocated to each node along the path of the video streams, we are assuming all nodes have the same service rate. The results obtained were based on a sample between 1 million and 10 million packets. The confidence intervals for the end-to-end delay percentile were computed using the batch means method. They are not given in the figures since they are extremely small.

\subsection{CISCO Telepresence}

The CISCO Telepresence trace has a lag-1 autocorrelation $\rho=-0.1383, c^{2}=4.40$ and an arrival rate $\lambda=7.34$ Mbps. The distribution of the packet sizes and the arrival rate (Mbps) are given in Figure 1. We observe that the packet size varied from $60 \mathrm{~B}$ to $1260 \mathrm{~B}$, and the arrival bit rate is almost constant. The almost constant arrival rate indicates that there are no scene changes in the video, which is normal for teleconferences.

We obtained results by varying the number $n$ of multiplexed traces from 1 to 30 . We generated the multiplexed traces synthetically in code. Each time, the trace was started at a random time uniformly distributed within the window of 0 to 25 seconds, so that to avoid temporal synchronization of the traces..

Table 1 gives some statistics regarding the $n$ multiplexed traces, for $n=1,10,20$, and 30 . We observe that the autocorrelation is zero. Also, the burstiness remains constant as the number of streams increase. This makes sense because the arrival rate of the Telepresence trace is almost constant, hence multiplexing it multiple times does not create any additional burstiness.
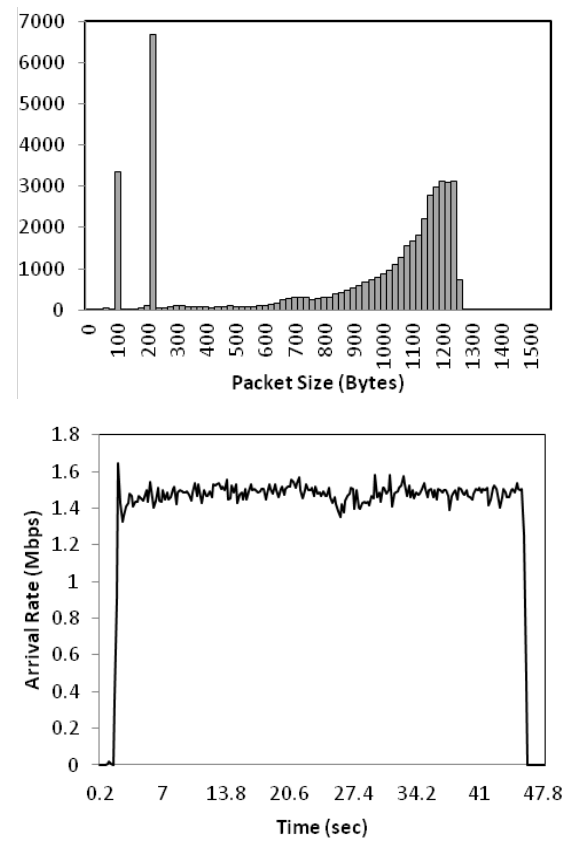

Figure 1. Telepresence Trace. Packet Length Distribution (top), Arrival Bit Rate (bottom).

Table 1. Statistics of Multiplexed Telepresence Traces.

\begin{tabular}{cccc}
\hline $\begin{array}{c}\text { Number of } \\
\text { Streams }(n)\end{array}$ & $\begin{array}{c}\text { Arrival Rate }(\lambda) \\
\text { Mbps }\end{array}$ & $\begin{array}{c}\text { Autocorrelation } \\
(\rho)\end{array}$ & $\begin{array}{c}\text { Burstiness } \\
\left(c^{2}\right)\end{array}$ \\
\hline 1 & 7.34 & -0.1383 & 4.40 \\
10 & 73.2 & -0.0938 & 3.15 \\
20 & 146 & -0.0477 & 3.60 \\
30 & 220 & -0.0582 & 3.43 \\
\hline
\end{tabular}


The results obtained, labeled "required bandwidth", are given in Figures $\mathbf{2}$ and 3. In addition, we plotted the average arrival rate of the multiplexed stream, labeled "average bandwidth", and the bandwidth obtained by multiplying the required bandwidth of a single stream, $R$, times the number of streams, $n$. labeled "no statistical gain". This is the bandwidth required assuming that no statistical gain is obtained by multiplexing $n$ streams i.e., the $n \times R$ values.

The two figures correspond to the two experimental conditions where the end-to-end percentile delay was fixed to be $30 \mathrm{~ms}$ and $50 \mathrm{~ms}$ respectively. We note some interesting properties. First, the average bandwidth required remains the same for all these cases. This is indeed the case as the average bandwidth required is the least amount of bandwidth required to keep the system stable and it solely depends on the average arrival rate. Second, as the end-to-end percentile delay constraint is relaxed (from 30 to 50) the required bandwidth curve becomes closer to the average bandwidth curve. Again this is intuitive as in order to satisfy tighter delay bounds (smaller values) we would require additional bandwidth than the average bandwidth. Similarly, if we keep relaxing the delay bounds, lesser bandwidth will be required to fulfill them.
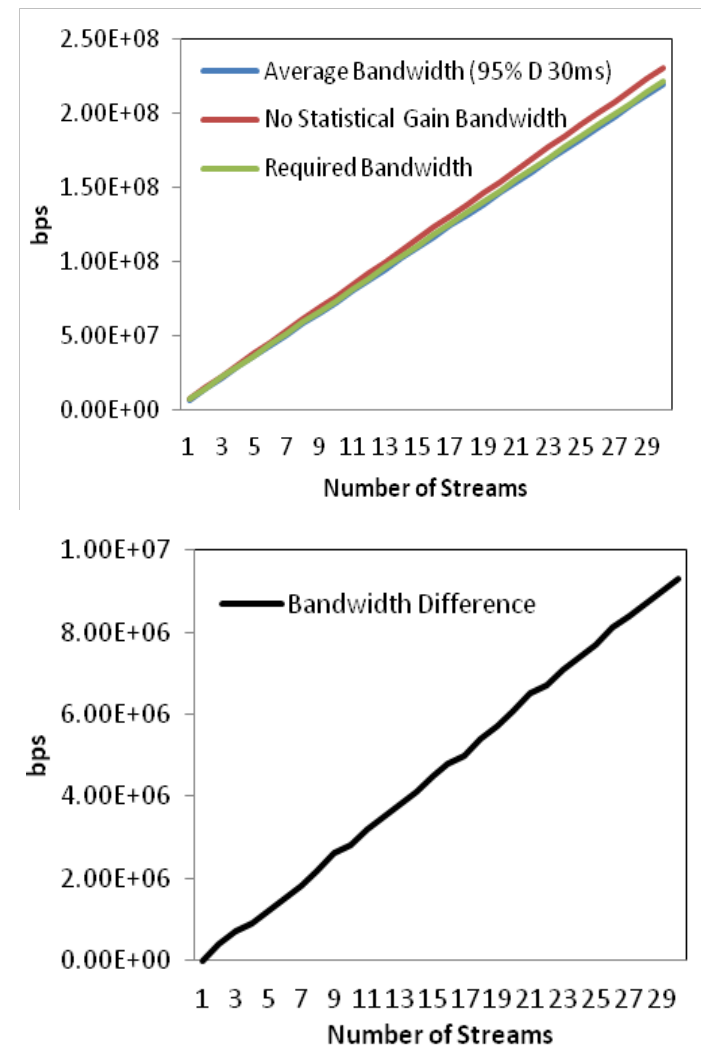

Figure 2. Bandwidth Requirement for Fixed 95th Percentile Delay of 30msec (top). Difference between No Statistical Gain and Required BW (bottom).
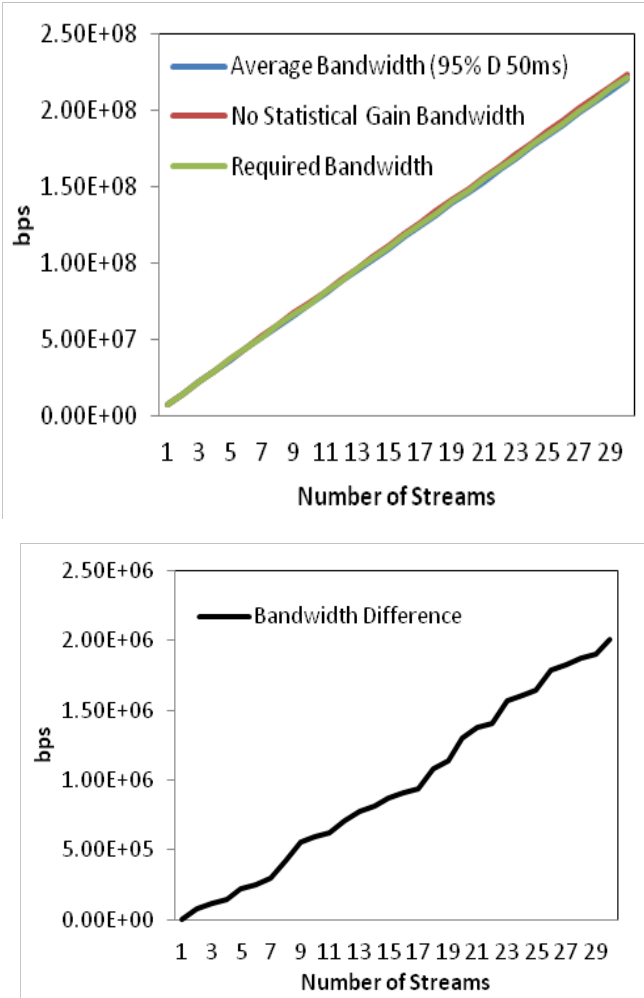

Figure 3. Bandwidth Requirement for Fixed 95th Percentile Delay of 50msec (top). Difference between No Statistical Gain and Required BW (bottom).

As can be seen, the required bandwidth is a linear function of the number of streams $n$. Infact, it is $c \times n \times R$, where $n$ is the number of streams, $R$ is the bandwidth requirement of a single flow and $c=0.96$ for Figure 2 and $c=0.99$ for Figure 3. It is also very close the case where no statistical gain is achieved, which is not surprising, since the transmission rate is almost constant due to lack of scene changes. The differences between the "required bandwidth" curve and the "no statistical gain" are plotted as "bandwidth difference" in a separate graph to highlight the linear difference.

Similar experiments were conducted for the interactive video stream of WebEx and the results generated were also the same. The graphs have been omitted in favor of the paper length.

\subsection{IPTV}

The IPTV trace has a lag- 1 autocorrelation $\rho=0.0579$, $c^{2}=3.6558$ and an arrival rate $\lambda=8.83$ Mbps. The distribution of the packet sizes and the arrival rate (Mbps) are given in Figure 4.

We observe that the trace had a small number of signaling packets, and the bulk of the traffic was video packets of size 1324B.

As before, we generated the multiplexed traces synthetically in code. Each time, we increased the number of 
concurrent streams from 1 to 30 where each stream is assigned a random starting point within the window of 0 to 15 seconds. This helps in preventing the temporal synchronization of the streams. Table 2 gives statistics for $n=1,10,20$, and 30 multiplexed streams.

The results obtained, labeled "required bandwidth", are given in Figures 5 and $\mathbf{6}$. In addition, we plotted the average arrival rate of the multiplexed stream, labeled "average bandwidth", and the bandwidth obtained by multiplying the required bandwidth of a single stream, $R$, times the number of streams, $n$, labeled "no statistical gain". The latter measure is the bandwidth required assuming that no statistical gain is obtained by multiplexing $n$ streams i.e., the $n \times R$ values.
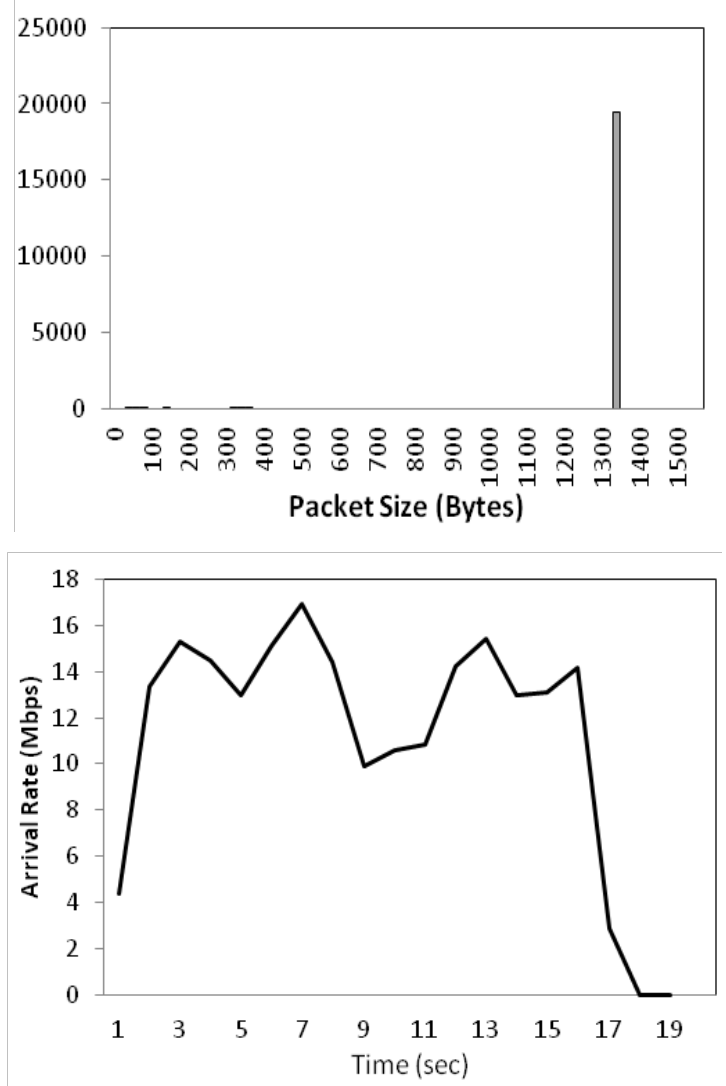

Figure 4. IPTV Trace. Packet Length Distribution (top), Arrival Bit Rate (bottom).

Table 2. Statistics of Multiplexed IPTV Traces.

\begin{tabular}{cccc}
\hline $\begin{array}{c}\text { Number of } \\
\text { Streams }(n)\end{array}$ & $\begin{array}{c}\text { Arrival Rate } \\
(\lambda) \text { Mbps }\end{array}$ & $\begin{array}{c}\text { Autocorrelation } \\
(\rho)\end{array}$ & $\begin{array}{c}\text { Burstiness } \\
\left(c^{2}\right)\end{array}$ \\
\hline 1 & 8.83 & 0.0579 & 3.65 \\
10 & 88.3 & -0.1686 & 2.54 \\
20 & 177 & -0.1011 & 2.36 \\
30 & 265 & -0.1004 & 3.31 \\
\hline
\end{tabular}

The two figures, Figures 5 and $\mathbf{6}$, correspond to the two experimental conditions where the end-to-end percentile delay was fixed to be $1 \mathrm{sec}$ and $3 \mathrm{sec}$ respectively. We note similar interesting properties as above for Telepresence (and for similar reasons). First, the average bandwidth required remains the same for all the cases. And second, as the end-to-end percentile delay constraint is relaxed (from $1 \mathrm{sec}$ to $3 \mathrm{sec}$ ) we see that the required bandwidth curve becomes closer to the average bandwidth curve.

As can be seen, the required bandwidth is a again a linear function of the number of streams $n$. Infact, it is $c \mathrm{x}$ $n \times R$, where $n$ is the number of streams, $R$ is the bandwidth requirement of a single flow and $c=0.95$ for Figure 5 and $c=0.956$ for Figure 6. The differences between the "required bandwidth" curve and the "no statistical gain" are plotted as "bandwidth difference" in a separate graph to highlight the linear difference.

\section{Verification of Linear Bandwidth Gain Using Queueing Theory Models}

The linearity behavior of bandwidth gain came as a surprise as it was expected that the gain will increase atleast as a second degree polynomial as the number of homogeneous streams increases. To make sure that this is not an aberration of the traces that we are using, we devised an independent experiment to validate this behavior.
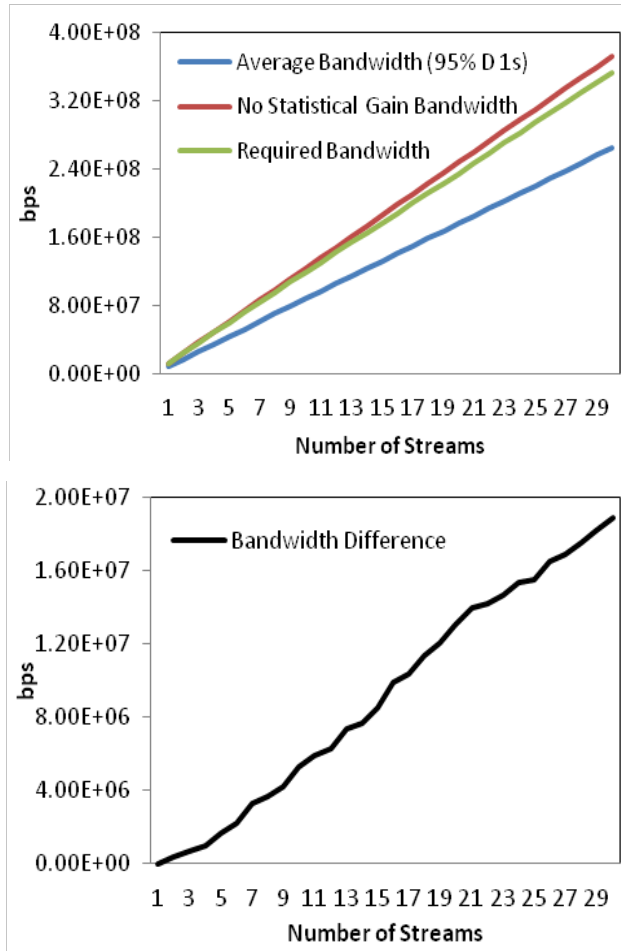

Figure 5. Bandwidth Requirement for Fixed 95th Percentile Delay of 1 sec (top). Difference between No statistical Gain and Required BW (bottom). 
An Interrupted Poisson Process (IPP) modulates between two exponentially distributed states, an $\mathrm{ON}$ and an OFF state. The transition rates for the ON and OFF states are respectively $\alpha$ and $\beta$, see Figure 7. While in the ON state, the process generates Poisson arrivals with rate $\lambda$ whereas in the OFF state there are no arrivals.

The aggregate of $n$ homogeneous IPPs (with parameters $\alpha, \beta$ and $\lambda$ ) is the well known Markov Modulated Poisson Process (MMPP) with $n+1$ states. This aggregate process of $n$ homogenous IPPs is given in Figure 8.
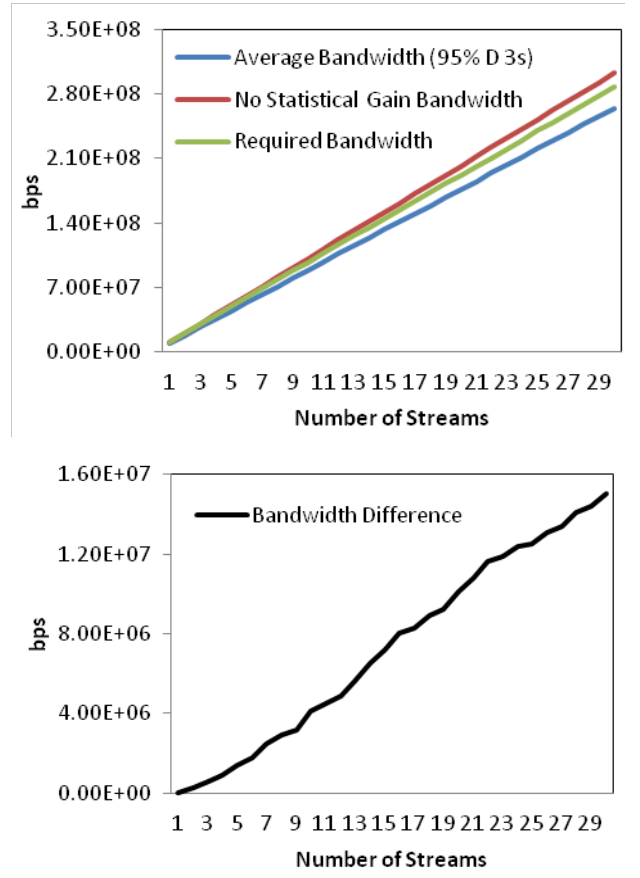

Figure 6. Bandwidth Requirement for Fixed 95th Percentile Delay of 3 sec (top). Difference between No statistical Gain and Required BW (bottom)

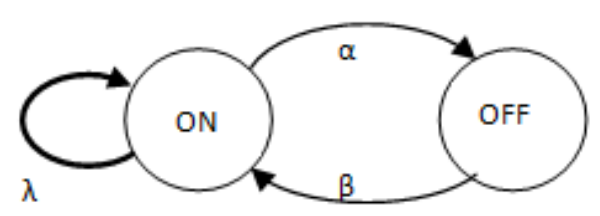

Figure 7. Markov Chain of an IPP.

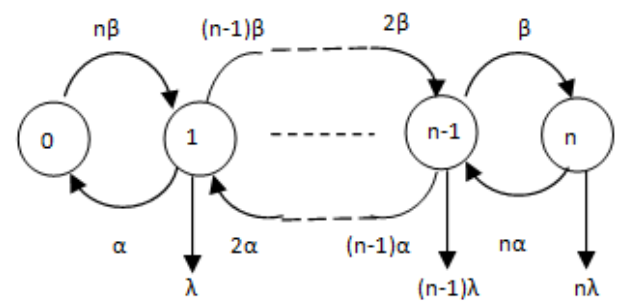

Figure 1. Markov Chain of an $(n+1)$ state MMPP, representing $n$ aggregate homogeneous IPPs
An IPP effectively models a VoIP call characteristics, i.e., voice spurts and silent periods. So, we can use an IPP to represent one VoIP call, or in other words, one stream. Next, we want to multiplex similar VoIP calls together. This will effectively mean multiplexing the same IPP multiple times. It is a well known result in Queueing theory that multiplexing $n$ IPPs results in the n-state MMPP process. Hence we use the resultant MMPP to represent multiplexed IPP streams.

The parameter values chosen for the IPP stream are set equal to the parameter values of a VoIP call. A VoIP has, on average, $400 \mathrm{~ms}$ of ON period, $600 \mathrm{~ms}$ of OFF period [6], and the number of packets generated per second during the ON period is 50. A voice payload of 160 bytes is carried in a separate IP packet with a 40-byte IP/ UDP/RTP header. Thus, we have $\alpha=2.5, \beta=1.667$ and $\lambda=50$. The QoS constraint enforced is that the $95^{\text {th }}$ percentile delay should be less than equal to $50 \mathrm{msec}$.

As can be seen in Figure 9, once again the required bandwidth is a linear function of the number of streams $n$. Infact, it is $c \times n \times R$, where $n$ is the number of streams, $R$ is the bandwidth requirement of a single flow and $c=$ 0.06 . The differences between the "required bandwidth" curve and the "no statistical gain" are plotted as "bandwidth difference" to highlight the linear difference between curves. Interestingly, we see a huge gain in bandwidth
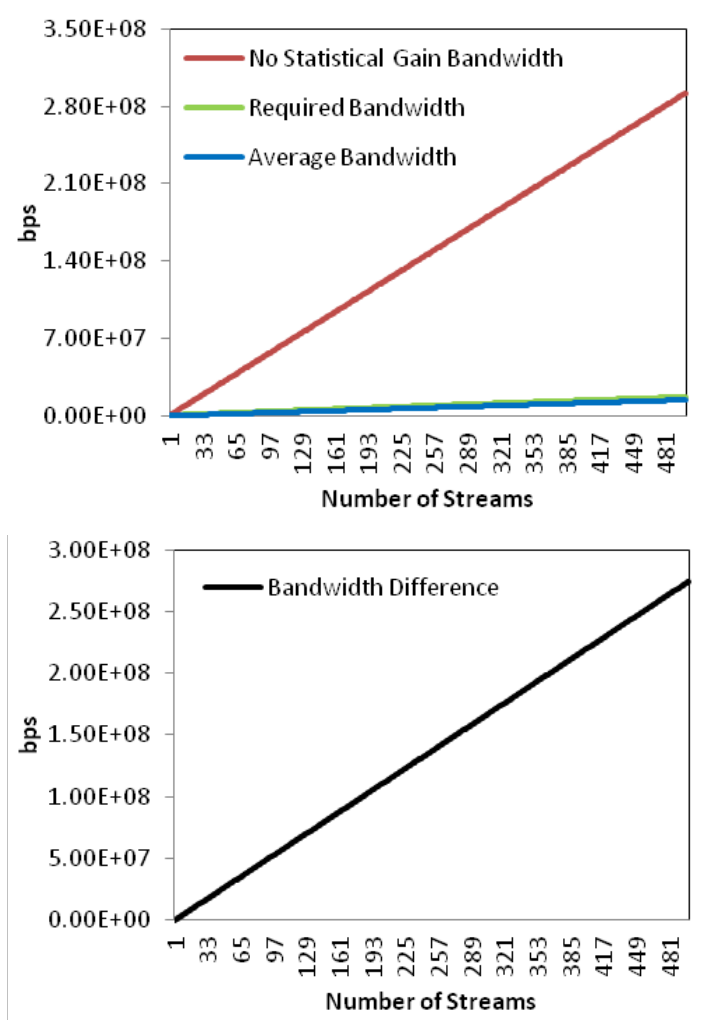

Figure 9. Bandwidth Requirement for Fixed 95th Percentile Delay of 50 msec (top). Difference between No statistical Gain and Required BW (bottom) 
here. The bandwidth gain increases as burstiness of original traffic increases and as arrival rate of the original traffic decreases, but, it retains its linear characteristic. An IPP is highly bursty because the arrivals are batched together during the ON period and no arrivals occur during the OFF period.

We would like to mention here that the linearity behavior has been independently confirmed by Lone [7].

\section{Validation of Linear Bandwidth Gain Using Algorithmic Asymptotic Analysis}

The linear behavior can also be explained by the algorithmic asymptotic analysis. For this, we will use the definition of big-theta $\Theta$ which implies asymptotic "equality". Before we proceed we need to define big-oh $\mathrm{O}$ and big-omega $\Omega$. The following definitions and Figure 10 are taken from [8].

Definition of big-oh: Let $f$ and $g$ be functions from the set of integers (or the set of real numbers) to the set of real numbers. Then $f(n)$ is said to be $\mathrm{O}(g(n))$, if and only if there are constants $C$ and $n_{0}$ such that

\section{$|f(n)| \geq C|g(n)|$, whenever $n>n_{0}$}

Big-oh is concerned with the "less than or equal to" relation between functions for large values of the variable. It is also possible to consider the "greater than or equal to" relation (big-Omega) and "equal to" relation (big-theta) in a similar way.

Definition of big-omega: $f(n)$ is said to be $\Omega(g(n))$, if there are constants $C$ and $n_{0}$ such that

$|f(n)| \leq \mathrm{C}|g(n)|$, whenever $n>n_{0}$

Definition of big-theta: $f(n)$ is said to be $\Theta(g(n))$, if $f(n)$ is $\mathrm{O}(g(n))$ and $f(n)$ is $\Omega(g(n))$. We also say that $f(n)$ is of order $g(n)$ or that both $f$ and $g$ are of the same order. $f(n)=\Theta(g(n))$ implies: $f(n)$ “=” $g(n)$. Formally, $\Theta(g(n))=$ $\left\{f(n)\right.$ : there exist positive constants $c_{1}, c_{2}$ and $n_{0}$ such that, $0 \leq c_{1} g(n) \leq f(n) \leq c_{2} g(n)$ for all $\left.n \geq n_{0}\right\}$.

This is represented pictorially in Figure 10, i.e., $g(n)$ is an asymptotically tight bound for $f(n)$.

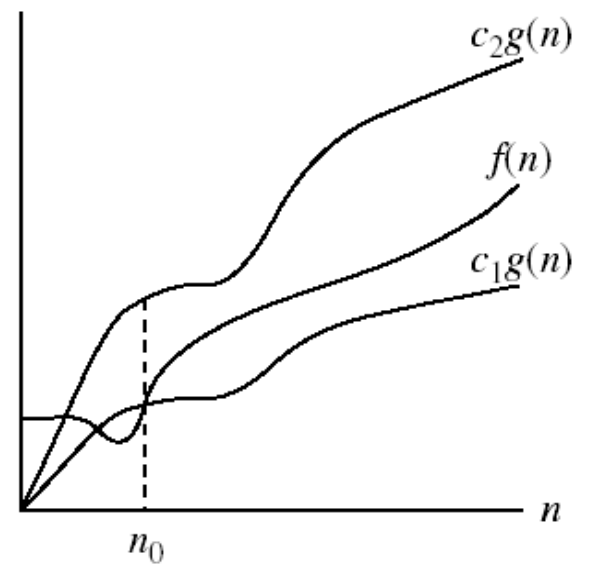

Figure 10. Pictorial Representation of big-theta.
In all four figures (Figures 2-5), the required bandwidth curve is bounded by the average bandwidth curve from below and the no statistical gain curve from above. The average bandwidth curve is linear, since the average bandwidth of $n$ streams is $n$ times the average bandwidth of a single stream. Similarly the no statistical gain (or maximum bandwidth) curve is linear and for the same reason.

Let us name the required bandwidth curve as $f(n)$, where $n$ is the number of streams, and let us define a function $g(n)=n$. We can define the average bandwidth curve by the straight line $g_{1}(n)=m_{1} n$, where $m_{1}$ is the average bandwidth required for a single stream. Similarly, the no statistical gain curve can be defined by the straight line $g_{2}(n)=m_{2} n$, where $m_{2}$ is the bandwidth required for a single stream. Now we have all the components of the big-theta definition, i.e.,

$0 \leq g_{1}(n) \leq f(n) \leq g_{2}(n)$ for all $n \geq 0$ or

$0 \leq m_{1} n \leq f(n) \leq m_{2} n$ for all $n \geq 0$ or

$0 \leq m_{1} g(n) \leq f(n) \leq m_{2} g(n)$ for all $n \geq 0$

Hence by definition of big-theta, $f(n)=\Theta(g(n))$ or $f(n)$ “=” $g(n)$. As $g(n)$ is a linear function, hence $f(n)$ will also be a linear function.

Thus we deduce that if a function $f(n)$ is bounded from above by a linear function and also from the below by a linear function, then it also behaves in a linear fashion.

\section{Conclusion}

In this paper, we first presented the generalized result that the statistical gain of combining homogeneous traffic streams, of any traffic type, is a linear function of the number of streams being multiplexed, for a fixed QoS constraint like percentile delay. We then presented linear bandwidth gain results for actual video traces, streaming video (IPTV traces) and interactive video (CISCO Telepresence traces). The linear bandwidth gain result is further verified using IPP and MMPP modeling and Big-O asymptotic analysis.

\section{REFERENCES}

[1] G. Van der Auwera, "Implications of Smoothing on Statistical Multiplexing of H.264/AVC and SVC Video Streams," IEEE Transactions on Broadcasting, vol. 55, no. 3, pp. 541-558, Sep. 2009.

[2] K. Angrishi, "Analysis of a Real-Time Network using Statistical Network Calculus with Effective Bandwidth and Effective Capacity," in 14th GI/ITG Conference on Measuring, Modeling and Evaluation, 2008, pp. 1-15.

[3] H. Li, C. Huang, and M. Devetsikiotis, "A Robust Adaptive Effective Bandwidth Allocation Technique,” in IEEE International Conference on Communications, 2005, pp. 115-119.

[4] K. Ravindran, M. Rabby, and X. Liu, "Bandwidth measurement and management for end-to-end connectivity 
over IP networks," in First International Communication Systems and Networks and Workshops, 2009, pp. 1-8.

[5] J. Jeong, S. Jeon, Y. H. Jung, and Y. Choe, "Statistical Multiplexing using scalable video coding for layered multicast," in IEEE International Symposium on Broadband Multimedia Systems, 2009, pp. 1-5.

[6] H. G. Perros, Connection-Oriented Networks: SONET/ SDH, ATM, MPLS, and OPTICAL NETWORKS, Wiley,
2005.

[7] Q. B. Lone, "Bandwidth Allocation for Video Streams Subject To An End-to-End Percentile Delay," MS Thesis, North Carolina State University, 2011.

[8] T. H. Cormen, C. E. Leiserson, R. L. Rivest, and C. Stein, Introduction to Algorithms, 3rd ed. Massachusetts Institute of Technology, 2009. 